\title{
INTERDISCIPLINARIDADE NO TRATAMENTO DE CERATOCISTO ODONTOGÊNICO: RELATO DE CASO
}

\author{
INTERDISCIPLINARITY IN THE TREATMENT OF ODONTOGENIC KERATOCYST: CASE REPORT
}

\author{
Thalles Eduardo RIBEIRO'; Dayane Batista SANTANA'; Sérgio Vitorino CARDOSO2; Luis Fernando Barbosa de PAUL03; \\ Claudia Jordão SILVA4; João César Guimarães HENRIQUES ${ }^{5}$ \\ Graduando em Odontologia, Faculdade de Odontologia, Universidade Federal de Uberlândia. \\ Professor Associado da Área de Patologia, Faculdade de Odontologia, Universidade Federal de Uberlândia. \\ Estomatologista do Hospital Odontológico/Hospital do Câncer da Universidade Federal de Uberlândia. Coordenador e Tutor do Programa de \\ Residência Multiprofissional em Oncologia. \\ Professora Adjunto II da Área de Cirurgia, Faculdade de Odontologia, Universidade Federal de Uberlândia. \\ Professor Adjunto I da Área de Diagnóstico Estomatológico, Faculdade de Odontologia, Universidade Federal de Uberlândia.
}

\section{RESUMO}

O Ceratocisto Odontogênico (CO) é um cisto de desenvolvimento odontogênico relativamente prevalente na população e com caráter eventual de agressividade com crescimento ântero-posterior insidioso. Trata-se de uma lesão cuja apresentação é extremamente diversa com relação à faixa etária, gênero e aspectos imaginológicos mais prevalentes. O presente trabalho relata o caso de um garoto de 11 anos de idade, portador de uma extensa lesão mandibular, envolvendo desde o dente 33 até o 46, cuja microscopia foi compatível com CO. O artigo aborda toda a conduta propedêutica conduzida pela equipe a nível ambulatorial, bem como a interdisciplinaridade com a ortodontia, possibilitando um desfecho almejado e satisfatório do caso.

PALAVRAS-CHAVE: Cistos odontogênicos; Ortodontia; Medicina oral.

\section{INTRODUÇÃO}

Dentre as lesões que acometem o complexo maxilo-mandibular, as lesões císticas merecem um especial destaque em virtude da prevalência e eventual morbidade que podem determinar aos indivíduos eventualmente acometidos ${ }^{1}$.

No contexto dos cistos odontogênicos de desenvolvimento, o Ceratocisto Odontogênico consiste em uma entidade de grande importância devido ao seu potencial de crescimento ântero-posterior e alta taxa de recorrência ${ }^{2}$.

A etiologia desta lesão ainda não é completamente entendida, havendo duas hipóteses possíveis, a origem por meio de remanescentes da lâmina dentária, e/ou a partir da proliferação de células da camada basal do epitélio oral da mandíbula e da maxila ${ }^{3-5}$.

A definição demográfica e morfológica do Ceratocisto Odontogênico é sempre desafiadora, uma vez que este cisto apresenta-se em indivíduos de variadas idades ${ }^{6,7}$ e com formas imaginológicas variadas ${ }^{8,9}$, indo desde as lesões unicísticas de menores dimensões até as lesões multicísticas de grande porte. Dessa forma, enucleações simples ${ }^{10}$ e marsupializações ${ }^{11}$ são opções terapêuticas viáveis e factíveis para resolução destas entidades e o complemento pela terapia ortodôntica por vezes se faz necessária, em decorrência de grandes deslocamentos dentários que comumente ocorrem ${ }^{5}$.

\section{RELATO DE CASO}

Paciente A.G.F, gênero masculino, 11 anos de idade, melanoderma, em bom estado de saúde geral compareceu, acompanhado pela mãe, ao ambulatório de Estomatologia da Faculdade de Odontologia da Universidade Federal de Uberlândia referenciado por um cirurgião-dentista. A responsável relatou que, após consulta odontológica de rotina, foi constatado um aumento de volume na região anterior direita da mandíbula. O exame extraoral mostrou discreta assimetria facial e na oroscopia foi possível perceber um abaulamento inferior do lado direito, junto dos dentes 83, 44 e 45 (Figura 1A, 1B e 1C). No exame radiográfico panorâmico inicial que o paciente portava, havia a presença de uma extensa imagem radiolúcida unilocular, com bordas bem delimitadas, envolvendo desde a raiz mesial do dente 33 até a raiz mesial do dente 46. Associado a lesão, o elemento 43 encontrava-se deslocado para a borda inferior mandibular e percebíamos o elemento 83 com rizólise avançada. Para melhor avaliação e detalhamento lesional, foram solicitadas uma radiografia oclusal e uma tomografia computadorizada de feixe cônico. Os exames de imagem elucidaram a expansão vestíbulo-lingual provocado pela lesão, apresentando mais de $4 \mathrm{~cm}$ em seu maior diâmetro e o afinamento da cortical basilar adjacente (Figura 2A, 2B e 2C).

Diante disso, foi realizada uma punção aspirativa da lesão, sendo esta positiva para um líquido amarelo-citrino, compatível com líquido cístico associado a grânulos esbranquiçados sugestivos de queratina. Sendo assim, a hipótese de diagnóstico sugerida foi a de Ceratocisto Odontogênico. No mesmo momento da punção aspirativa, um fragmento da cápsula lesional 
foi removido para exame histopatológico e uma descompressão lesional conduzida com vistas a uma regressão progressiva do provável extenso cisto, previamente à posterior enucleação. No corte histopatológico foi evidenciado uma lesão cística neoplásica, constituída por cápsula de tecido conjuntivo propriamente dito, denso, não modelado, permeado discretamente por hemácias e discretos fragmentos ósseos. O tecido epitelial de revestimento se apresentava uniformemente em espessura, não apresentando cones epiteliais evidentes, constituído por poucas camadas celulares, mostrando camada superficial de paraqueratose corrugada, e camada basal cujos queratinócitos colunares apresentavam núcleos hipercromáticos com disposição em paliçadas. Sendo o laudo compatível com Ceratocisto Odontogênico (Figura 3).

A mãe, na presença do paciente, foi orientada a realizar irrigações 2 vezes ao dia com solução de clorexidina $0,2 \%$ para favorecer o processo de redução lesional e a limpeza da cavidade. O paciente seguiu acompanhamento clínico mensal e, decorridos 3 meses, novos exames radiográficos periapical e panorâmico demonstraram neoformação óssea centrípeta e deslocamento do dente 43 da base da mandíbula em direção a crista óssea correspondente. Seis meses após o primeiro atendimento, a equipe de estomatologia em comum acordo com o ortodontista do paciente conduziram a colagem de bracket no elemento 44 e a colagem de botão ortodôntico no dente 43 para tracionamento do mesmo.

Transcorridos 8 meses de terapia ortodôntica foi possível finalizar o correto posicionamento do dente 43 , mesmo com certo grau de dilaceração radicular, e conduzir uma curetagem final do que ainda havia de remanescente do ceratocisto submetido à descompressão. O tratamento foi satisfatoriamente finalizado e o paciente segue em proservação clínica e imaginológica trimestral (Figura 4A e 4B).

\section{DISCUSSÃO}

O Ceratocisto Odontogênico é uma lesão intraóssea dos ossos gnáticos ${ }^{12,13}$ relativamente comum e frequentemente desafiadora para os profissionais da odontologia. A ampla gama de apresentações nos ossos maxilares ${ }^{14,15}$, incidindo em faixas
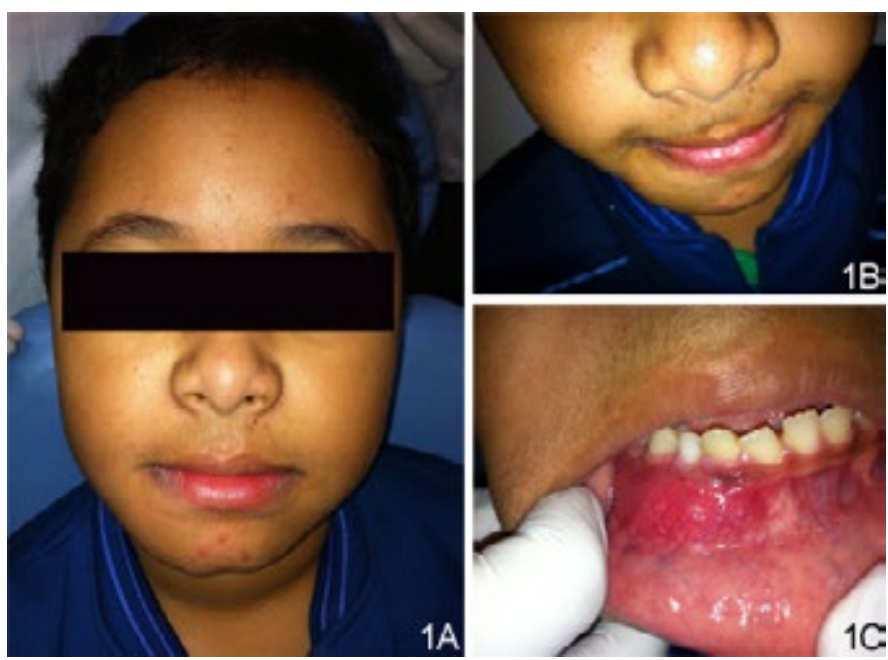

$1 \mathrm{~A}$

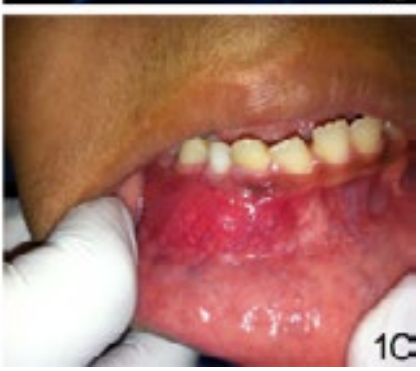

Figura 1 - (A) Discreta assimetria no exame extraoral. (B) Abaulamento inferior direito. (C) Tumefação visualizada intraoralmente
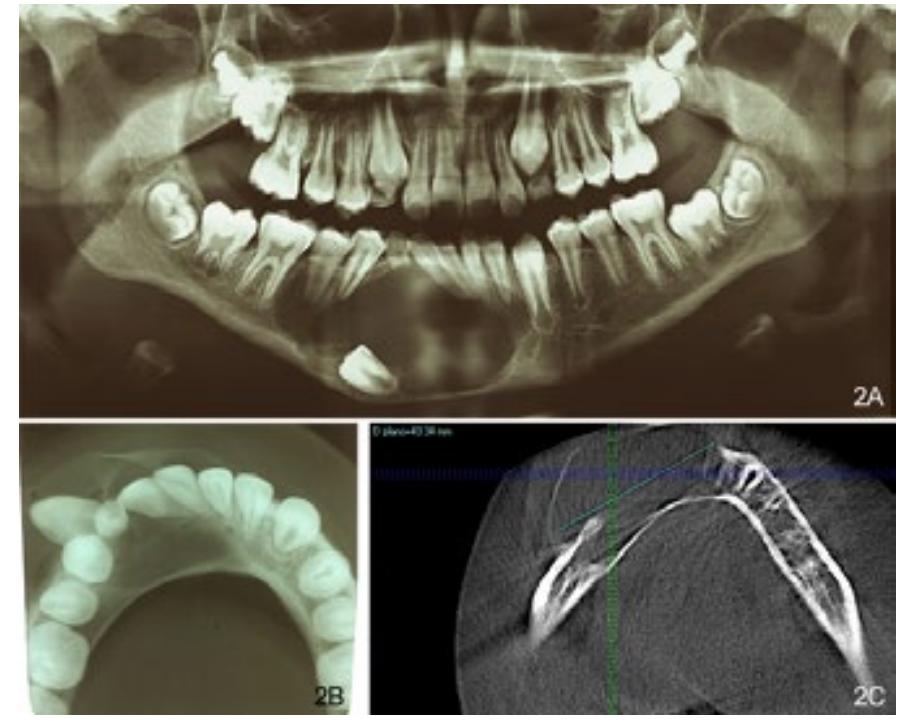

Figura 2 - (A) Radiografia panorâmica mostrando a extensão lesional com deslocamento basilar do dente 43. (B) Expansão vestíbulo-lingual mostrada na radiografia oclusal. (C) Corte axial tomográfico mostrando diâmetro de mais de $4 \mathrm{~cm}$

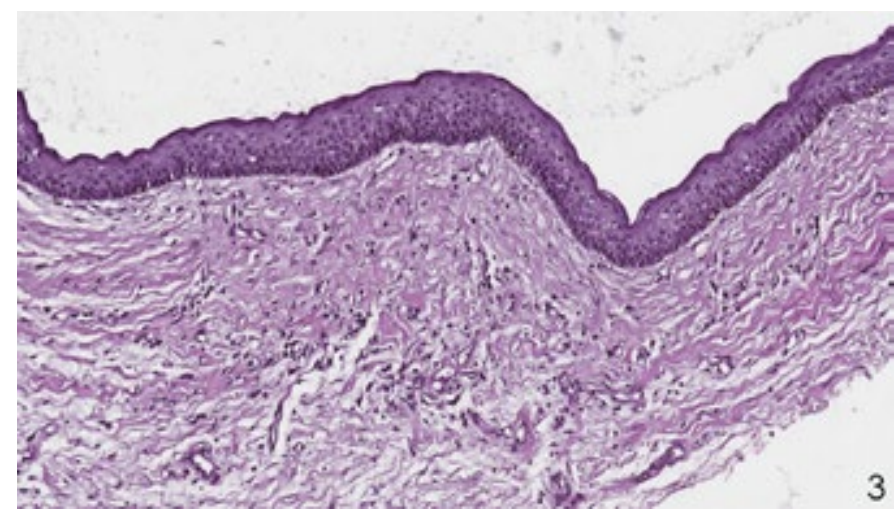

Figura 3 - Microscopia mostrando paraqueratose corrugada e queratinócitos colunares hipercromáticos em paliçada
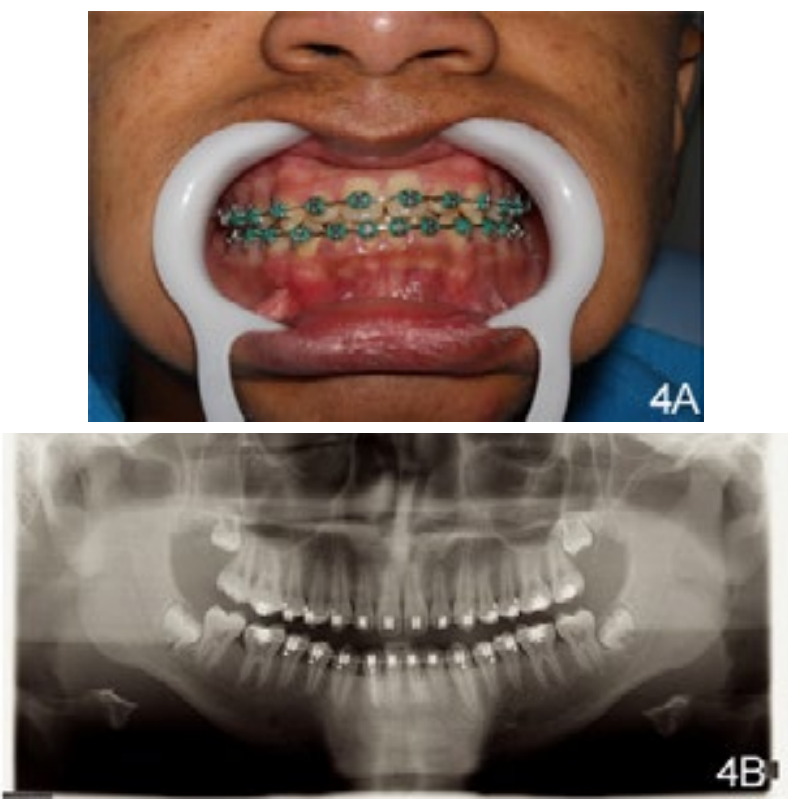

Figura 4 - (A/B) Visão frontal em oclusão do paciente após 8 meses de terapia ortodôntica 
etárias variadas ${ }^{10,16,17}$, determina com que haja desde lesões pequenas de fácil tratamento até lesões extensas, eventualmente mostrando difícil resolução. Neste sentido, o curso preferencialmente ântero-posterior desta lesão faz com que, em muitos casos, o diagnóstico seja tardio piorando por vezes o prognóstico e o tratamento ${ }^{2}$.

No presente caso, o cirurgião-dentista do paciente percebeu um abaulamento na região sinfisária justificando o referenciamento do mesmo para um serviço especializado. Como era de se esperar para um ceratocisto, a lesão já mostrava extensa dimensão elucidada pela radiografia panorâmica ${ }^{2}$. Felizmente, apesar do caráter agressivo de desenvolvimento e progressão ${ }^{7}$, o ceratocisto odontogênico responde usualmente de forma positiva aos processos descompressivos ${ }^{19,20}$. Sendo assim, a associação de uma descompressão adequada aliada à cooperação da família, por meio dos cuidados dispensados pela mãe especialmente quanto às irrigações diárias, além da tenra idade do paciente, permitiram uma rápida melhora representada pela progressiva diminuição lesional, com progressiva neoformação óssea e sem que houvesse a perda de nenhum elemento dentário associado $^{21}, 22$.

Finalmente, é fundamental que abordemos a importância da especialidade ortodontia na complementação terapêutica das lesões intraósseas dos maxilares ${ }^{5}$. A estreita comunicação interdisciplinar entre estomatologista e ortodontista, presente no relato apresentado, foi condição sine qua non para o devido êxito do tratamento obtido. Desde a primeira intervenção ortodôntica, houve comunicações entre os profissionais que permitiram o devido conhecimento das condutas por parte do ortodontista responsável, permitindo ao mesmo a segurança necessária para que pudesse conduzir os posicionamentos dentários de forma apropriada em prol do restabelecimento estético e funcional do paciente, sem subtração de nenhum elemento dentário ${ }^{23}$. $\mathrm{O}$ trabalho ora descrito elucida o quanto a odontologia pode ser eficaz e benéfica aos pacientes quando desenvolvida com responsabilidade, conhecimento científico e especialmente de forma integrada às diversas especialidades que a compõem, enquanto nobre área da medicina humana.

\section{CONCLUSÃO}

O Ceratocisto Odontogênico é uma lesão que, apesar de benigna, exige atenção e conhecimento por parte dos cirurgiões-dentistas dada sua prevalência relativamente elevada e eventual agressividade.

Assim como em qualquer área da saúde humana, a interdisciplinaridade na estomatologia é essencial para uma adequada resolutividade de casos, sendo a ortodontia uma aliada comumente essencial.

\section{REFERÊNCIAS}

01. Shear M. Speight's Cysts of Oral and maxillofacial regions. Blackwell Munksgaard; 2007.

02. Pazdera V, Kolar Z, Zboril V, Tvrdy P, Pink R. Odontogenic keratocysts/keratocystic odontogenic tumours: biological characteristics, clinical manifestation and treatment. Biomed Pap Med Fac Univ Palacky Olomouc Czech Repub. 2014; 158(2):170-174.

03. Marques JAF, Neves JL, Alencar DA, Lemos IM, Marques LC. Ceratocisto odontogênico: relato de caso. Sitientibus. 2006; 34: 59-69.
04. Lopes MWF, Souza GFM, Carvalho EJA, Gondola AO. Aspectos clínico-morfológicos do queratocisto odontogênico: relato de caso. Odontol Clín Cient. 2004; 3(1):61-5.

05. Peixoto RF, Menezes DPB, Pereira JSP, Pereira KMA, Costa ALL. Tumor odontogênico ceratocístico: revisão dos achados atuais. Rev Cir Traumatol Buco-Maxilo-fac. 2009; 9(3):21-8.

06. Rezende FCB, Dias MA. Tumor Odontogênico Queratocisto: relato de caso. Rev Eletrônica Acervo Saúde. 2016; 8(4): 938-941.

07. Wright JM, Vered M. Update from the 4th Edition of the World Health Organization Classification of Head and Neck Tumours: Odontogenic and Maxillofacial Bone Tumors. Head Neck Pathol. 2017; 11(1):68-77.

08. Deyhimi P, Hashemzadeh Z. Study of the biologic behavior of odontogenic keratocyst and orthokeratinzaided odontogenic cyst using TGF-alpha and P53 markers. Pathol Res Pract. 2014; 210(4):201-4.

09. Aciole GTS, Santos MAM, Aciole JMS, Ribeiro Neto N, Pinheiro ALB. Tumor odontogênico queratocisto recidivante: tratamento cirúrgico conservador ou radical? Relato de caso clínico. Rev Cir Traumatol Buco-Maxilo-fac. 2010; 10(1): 43-48.

10. Garcia CB, Gignon VF, Melo MR, Costa SAA, Costa JMC, Patrocínio LG. Tumor odontogênico queratocístico extenso de mandíbula: enucleação associada à crioterapia. Rev Bras Cir Craniomaxilofac. 2011; 14(1):60-2.

11. Neville BW, Damm DD, Allen CM, Bouquot JE. Patologia oral e maxilofacial.. Rio de Janeiro: Guanabara Koogan; 2004.

12. Harmon M, Arrigan M, Toner M, O'Keeffe SA. A radiological approach to benign and malignant lesions of the mandible. Clin Radiol. 2015; 70(4):335-50.

13. Borghesi A, Cosimo N, Giannito C, Tironi A, Maroldi R, Bartolomeo FD, Preda L. Odontogenic keratocyst: imaging features of a benign lesio with an agressive behaviour. Insights Imaging. 2018; 9(5):883-897.

14. MacDonald D. Lesions of the jaws presenting as radiolucencies on cone-beam CT. Clin Radiol. 2016; 71(10):972-85.

15. Ketheeswaranathan V, George R. A case report of an unsual presentation of a keratocystic odontogenic tumour in the anterior mandible. Dent Update. 2016; 43(9): 891-3.

16. Tekkesin MS, Tuna EB, Olgac V, Aksakalli, Alath C. Odontogenic lesions in a pediatric population: review of the literature and presentation of 745 cases. Int J Pediatr Otorhinolaryngol. 2016; 86:196-9.

17. Deboni MC, Brozoski MA, Traina AA, Acay RR, Naclério-Homem MG. Surgical management of dentigerous cyst and keratocystic odontogenic tumor in children: a conservative approach and 7-year follow-up. J Appl Oral Sci. 2012; 20(2):268-71.

18. Philipsen HP. Keratocystic odontogenic tumours. In: Barnes L, Eveson JW, Reichart P, Sindransky D. World Health Organization classification of tumours. Pathology and genetics of head and neck tumors. Lyon: IARC Press; 2005. p. 306-7.

19. Santos RSL, Ramos-Perez FMM, Silva GKA, Rocha AC, Prado JD, Perez DEC. Odontogenic keratocyst: The role of the orthodontist in the diagnosis of initial lesions. Am J Orthod Dentofacial Orthop. 2017; 152(4):553-556.

20. Kinard BE, Chuang SK, August M, Dodson TB. For treatment of odontogenic keratocysts, is enucleation, when compared to decompression, a less complex management protocol? J Oral Maxillofac Surg. 2015; 73(4):641-8. 
21. Alpy A, Tournaire L, Vaysse F, Marchal-Sixou C, Lhomme A, Courtois B. Interest of decompression in orthodontics: Case report of a keratocyst during childhood. Int Orthod. 2017; 15(2):238-250.

22. Allon DM, Allon I, Anavi Y, Kaplan I, Chaushu G. Decompression as a treatment of odontogenic cystic lesions in children. J Oral Maxillofac Surg. 2015; 73(4):649-54.
23. August M, Faquin WC, Troulis MJ, Kaban LB. Dedifferentiation of odontogenic keratocyst epithelium after cyst decompression. J Oral Maxillofac Surg. 2003; 61(6):678-83.

\section{ABSTRACT}

Keratocyst Odontogenic (KO) is a cyst of odontogenic development relatively prevalent in the population and with an eventual aggressive character with insidious antero-posterior growth. It is a lesion whose presentation is extremely diverse in relation to age, gender and most prevalent imaging aspects. This paper reports the case of an 11 - year - old boy with an extensive mandibular lesion, evolving from tooth 33 to 46, whose microscopy was compatible with $\mathrm{KO}$. The article deals with all the propaedeutic conduct conducted by the team at the outpatient level, as well as the interdisciplinarity with orthodontics, enabling a desired and satisfactory outcome of the case.

KEYWORDS: Odontogenic cyst; Ortodonthics; Oral medicine.

\section{AUTOR PARA CORRESPONDÊNCIA}

Thalles Eduardo Ribeiro

Avenida Pará, 1720, bairro Umuarama

E-mail: thalledu@hotmail.com 